\title{
Interactive comment on "Quantification and evaluation of atmospheric pollutant emissions from open biomass burning with multiple methods: A case study for Yangtze River Delta region, China" by Yang Yang and Yu Zhao
}

\section{Anonymous Referee \#2}

Received and published: 19 September 2018

This manuscript estimates the air pollutant emissions from open biomass burning (OBB) in Yangtze River Delta for 2005-2015 using traditional bottom-up, fire radiative power (FRP)-based, and constraining approaches, and analyzed the differences between those methods and their underlying reasons. The manuscript is generally well written. However, there are still some issues in the manuscript which authors shall pay attention to. So the paper cannot be accepted for publication before authors address the following comments.

1. As shown in Table S1 and Table S4, the authors use different emission factors for 
OBB in bottom-up method and FRP-based method. I suggest same emission factors shall be used for both methods. This is why that for most air pollutants, emissions ACPD estimated by bottom-up method is higher than that by FRP-based but the emissions of NMVOC and NH3 from bottom-up method is much lower than that by FRP-based method.

Interactive

2. The spatial resolutions of the two domains were set at 27 and $9 \mathrm{~km}$ respectively. $9 \mathrm{~km}$ is kind of coarse resolution. How does this spatial resolution affect the CMAQ modeling results? Will you get a better model performance if you use a $3 \mathrm{~km}$ resolution?

3. Considering that the PM emissions from OBB are mainly PM2.5, and the ambient PM10 is more affected by the local road dust emissions, it is not appropriate to only use PM10 concentration to evaluate the model performance and analyze the contribution of OBB. I think authors shall use both PM10, PM2.5, CO, NO2, SO2, OC, EC to do the model evaluation. At least PM2.5 shall be included considering that most Chinese cities release PM2.5 hourly concentrations since 2013. Although authors give a couple of figures in $\mathrm{SI}$, this is not enough. Specifically, the correction based on the comparisons of PM10 cannot be used for all other species.

4. The model performance statistics for meteorological parameters shown in Table S6 and that for PM10 concentrations as shown in Table 2 shall include the benchmark of the evaluation.

5. For OBB, temporal allocation is very important. It is good to see the monthly variations of fire occurrence in Figure 1. However, the authors only give information for year 2010 and 2012, I wonder if the authors can provide such information for other years.

6. Figure 2 shall give the name of each city in the YRD. Otherwise it is difficult for readers to understand when author talk about Lianyungang, Fuyang, Shanghai, Suzhou, Wuxi, Changzhou, etc.

Printer-friendly version

7. The color in Figure 4 is very difficult to read. 
Interactive comment on Atmos. Chem. Phys. Discuss., https://doi.org/10.5194/acp-2018-701, 2018.
ACPD

Interactive comment 